\title{
Weak Closure Theorem fails for $\mathbb{Z}^{2}$-actions
}

\author{
by \\ T. Downarowicz (Wrocław) and J. Kwiatkowski (Toruń)
}

\begin{abstract}
We construct an example of a Morse $\mathbb{Z}^{2}$-action which has rank one and whose centralizer contains elements which cannot be weakly approximated by the transformations of the action.
\end{abstract}

Introduction. The rank of a measure preserving transformation (i.e., of a $\mathbb{Z}$-action) was defined by B. V. Chacon in 1970 ([C]). Roughly speaking, rank is the minimal number of towers needed to approximate the sigmaalgebra, and it is an invariant of measure conjugacy. Chacon proved that rank is always not smaller than spectral multiplicity, which makes rank an important parameter. Whether rank is an invariant of spectral isomorphism is a famous, still unsolved, problem in ergodic theory.

Rank can also be defined for actions of other abelian groups whenever it is clear what we understand by towers. This is equivalent to a choice of a Fölner sequence of sets. For $\mathbb{Z}^{2}$ the most natural choice is rectangles (or parallelepipeds in $\mathbb{Z}^{d}$ ). With such choice Chacon's inequality still holds.

The metric centralizer of an abelian group $\mathbb{T}$ of measure preserving transformations is defined as the family of all automorphisms of the space which commute with all elements of $\mathbb{T}$. Endowed with the weak topology, it has the structure of a complete metric topological group and obviously it contains $\mathbb{T}$, hence also its closure $\mathrm{Wcl}(\mathbb{T})$. The centralizer can be viewed as a family of special self-joinings and hence has gained a lot of attention in ergodic theory (see e.g. [K2], [J-R]).

In 1986 J. King ([K1]) proved that for rank-one $\mathbb{Z}$-actions the centralizer coincides with $\mathrm{Wcl}(\mathbb{T})$ (see also $[R]$ for another proof). This theorem is

2000 Mathematics Subject Classification: 37A05, 37A15, 37A35.

Key words and phrases: $\mathbb{Z}^{2}$-action, weak closure theorem, rank one, metric centralizer, Morse sequence.

Research of the second author supported by the grant KBN 5 P03A 02721 . The first draft of this paper was written while the authors were visiting the Université de Bretagne Occidentale, March 2001. 
known as the Weak Closure Theorem and it has found many important applications. For example, in the class of Morse flows it allows one to prove that rank-one is in fact a spectral invariant ([D-K-L]). Whether an analogous Weak Closure Theorem holds for other group actions (in particular for $\mathbb{Z}^{d}$ actions) is a natural question (see e.g. [G-R]).

In this paper we construct an example of a rank-one $\mathbb{Z}^{2}$-action which does not have this property. Moreover, this will be done in the class of Morse flows. Our method admits a modification with the additional property that the system is rigid, which implies that $\mathrm{Wcl}(\mathbb{T})$ is uncountable.

The authors would like to acknowledge the hospitality of Département de Mathématiques, Université de Bretagne Occidentale in Brest, France, where this article was written.

Preliminaries and notation. Let $\mathbb{Z}^{2}$ denote the set of 2-dimensional integers $\bar{n}=\left(n_{1}, n_{2}\right), \overline{0}=(0,0), \overline{1}=(1,1)$. We will write $v(\bar{n})=\left|n_{1} n_{2}\right|$. In $\mathbb{Z}^{2}$ we introduce a partial order by $\bar{m} \leq \bar{n} \Leftrightarrow m_{1} \leq n_{1} \& m_{2} \leq n_{2}$. We shall say that a sequence $\left(\bar{n}_{k}\right)$ tends to infinity if both coordinates of $\bar{n}_{k}$ tend to infinity. For $\bar{m} \leq \bar{n}$ the rectangle $\{\bar{i}: \bar{m} \leq \bar{i} \leq \bar{n}\}$ will be denoted by $[\bar{m}, \bar{n}]$.

Let $G$ be a finite set. A finite $G$-valued matrix $B=(B(\bar{i}))_{\bar{i} \in[\overline{0}, \bar{n}-\overline{1}]}$ will be called a block of size $\bar{n}$. The size of $B$ will be denoted by $\bar{n}(B)$, and we will write $v(B)$ for $v(\bar{n}(B)$ ) (the number of entries of $B$ ).

For two blocks $B$ and $C$ of the same size $\bar{n}$ the Hamming distance is defined as

$$
\bar{d}(B, C)=\frac{1}{v(\bar{n})} \#\{\bar{i} \in[\overline{0}, \bar{n}-\overline{1}]: B(\bar{i}) \neq C(\bar{i})\} .
$$

The elements of $G^{\mathbb{Z}^{2}}$ and of $G^{\mathbb{N}^{2}}$ are called arrays and quarter-arrays, respectively. For an array (or quarter-array) $x=(x(\bar{n}))$ we denote by $x[\bar{m}, \bar{n}]$ the block $B=(x(\bar{i}))_{\bar{i} \in[\bar{m}, \bar{n}]}$. We then say that $B$ occurs in $x$ at position $\bar{m}$. Similarly, a block $B$ may occur in another block $C$ (larger in size). The Hamming distance between arrays or quarter-arrays $x$ and $y$ is defined as the upper limit of the distances of the blocks $x[-\bar{n}, \bar{n}]$ and $y[-\bar{n}, \bar{n}]$ (or $x[\overline{0}, \bar{n}]$ and $y[\overline{0}, \bar{n}]$ for quarter-arrays) as $\bar{n}$ tends to infinity.

We will consider the $\mathbb{Z}^{2}$-action of the group $\mathbb{T}=\left\{T^{\bar{n}}: \bar{n} \in \mathbb{Z}^{2}\right\}$ of shifts on $G^{\mathbb{Z}^{2}}$ defined by

$$
T^{\bar{n}} x(\bar{m})=x(\bar{m}+\bar{n})
$$

as a topological flow ( $G \mathbb{Z}^{2}$ is endowed with the product topology). We will restrict the phase space to the set $X_{x}$, where $x$ is a quarter-array, of arrays $y$ such that every block appearing in $y$ occurs in $x$ at infinitely many positions. (Clearly, $X_{x}$ is closed and $\mathbb{T}$-invariant.) We will be dealing mainly with strictly ergodic flows $\left(X_{x}, \mathbb{T}\right)$, i.e., such that $X_{x}$ is a minimal (nonempty) 
closed $\mathbb{T}$-invariant set and there exists a unique $\mathbb{T}$-invariant Borel probability measure $\mu$ on $X_{x}$.

Let $(X, \mu, \mathbb{T})$ be a measure preserving $\mathbb{Z}^{2}$-action represented in symbolic form, i.e., in the form of the shift action on arrays over a finite alphabet $G$. We will now specify what we mean by saying that $(X, \mu, \mathbb{T})$ is of rank one. The definition below is an equivalent interpretation of the abstract definition (involving towers along rectangles) in the case of symbolic systems.

Let $y$ be an array. We say that a block $B$-occurs in $y$ at position $\bar{m}$ if

$$
\bar{d}(B, y[\bar{m}, \bar{m}+\bar{n}(B)-\overline{1}]) \leq \varepsilon .
$$

We say that $B \varepsilon$-covers $y$ if there exists a subset $\mathbb{P} \subset \mathbb{Z}^{2}$ with the following properties:

- $[\bar{m}, \bar{m}+\bar{n}(B)-\overline{1}] \cap \mathbb{P}=\{\bar{m}\}$ for each $\bar{m} \in \mathbb{P}$,

- the density of $\mathbb{P}$ in $\mathbb{Z}^{2}$ is at least $(1-\varepsilon) / v(B)$,

- $B \varepsilon$-occurs in $y$ at every position $\bar{m} \in \mathbb{P}$.

Roughly speaking, some disjoint $\varepsilon$-occurrences of $B$ in $y$ cover nearly all of $\mathbb{Z}^{2}$. The system $(X, \mu, \mathbb{T})$ is said to be of rank one if for every $\varepsilon>0$ there exists a block $B_{\varepsilon}$ such that $\mu$-almost every $y \in X$ can be $\varepsilon$-covered by $B_{\varepsilon}$, and the blocks can be chosen so that $\bar{n}\left(B_{\varepsilon}\right)$ tends to infinity as $\varepsilon \rightarrow 0$.

By the centralizer $\mathcal{C}(X, \mu, \mathbb{T})$ (or briefly $\mathcal{C}(X, \mathbb{T})$ in strictly ergodic systems) of $(X, \mu, \mathbb{T})$ we mean the set of all automorphisms of the Borel measure space $(X, \mu)$ which commute with every element of the acting group $\mathbb{T}$. Obviously, $\mathbb{T} \subset \mathcal{C}(X, \mu, \mathbb{T})$. In $\mathcal{C}(X, \mu, \mathbb{T})$ we will consider the weak topology defined as follows:

$$
\begin{aligned}
S_{n} \rightarrow S \Leftrightarrow & \text { for every Borel set } F \subset X, \\
& \mu\left(S_{n}(F) \triangle S(F)\right) \underset{n \rightarrow \infty}{\longrightarrow} 0 \text { and } \mu\left(S_{n}^{-1}(F) \triangle S^{-1}(F)\right) \underset{n \rightarrow \infty}{\longrightarrow} 0 .
\end{aligned}
$$

It is known (see e.g. [K1]) that in the symbolic case $S_{n} \rightarrow S$ if and only if $\bar{d}\left(S_{n}(y), S(y)\right) \rightarrow 0$ and $\bar{d}\left(S_{n}^{-1}(y), S^{-1}(y)\right) \rightarrow 0$ for $\mu$-almost every $y \in X$. With this topology the centralizer has the structure of a complete metric topological group. We denote by $\operatorname{Wcl}(\mathbb{T})$ the closure of $\mathbb{T}$ in $\mathcal{C}(X, \mu, \mathbb{T})$.

Our next subject is the definition of a Morse $\mathbb{Z}^{2}$-action. From now on we will assume that $G$ is a finite abelian group (denoted multiplicatively) with unity $e$. If $g \in G$ then $B \cdot g$ is obtained by multiplying each entry of $B$ by $g$ (the rule for arrays is analogous). Let $B$ and $C$ be two blocks of symbols from $G$; set $\bar{n}=\bar{n}(B), \bar{m}=\bar{n}(C)$. The product $B \times C$ of these blocks is a block of size $\bar{m} \bar{n}=\left(m_{1} n_{1}, m_{2} n_{2}\right)$, defined by

$$
(B \times C)(\bar{s}+\bar{n} \bar{t})=B(\bar{s}) C(\bar{t})
$$

$(\bar{s} \in[\overline{0}, \bar{n}-\overline{1}], \bar{t} \in[\overline{0}, \bar{m}-\overline{1}])$. Clearly, $v(B \times C)=v(B) v(C)$. 
For a given sequence $\left(B_{q}\right)_{q \in \mathbb{N}}$ of blocks such that $\bar{n}_{q}=\bar{n}\left(B_{q}\right) \geq(2,2)$ and $B_{q}(\overline{0})=e$ for each $q$ we define the products

$$
A_{q}=B_{1} \times \ldots \times B_{q} .
$$

They have sizes $\bar{p}_{q}=\bar{n}_{1} \ldots \bar{n}_{q} \rightarrow \infty$ and these blocks converge at each coordinate to a quarter-array denoted by $A$ or $B_{1} \times B_{2} \times \ldots$ If $A$ happens not to be periodic in any direction, then it is called a Morse quarter-array. If $x=A$ is a Morse quarter-array then the symbolic $\mathbb{Z}^{2}$-action of $\mathbb{T}$ on $X_{x}$ is called a Morse $\mathbb{Z}^{2}$-action generated by the sequence $\left(B_{q}\right)$ of blocks. The proof of the following fact is analogous to the one-dimensional case, and will be omitted:

FACT 1. A sufficient condition for a Morse $\mathbb{Z}^{2}$-action generated by a sequence $\left(B_{q}\right)$ of blocks to be strictly ergodic is that there exists an $\varepsilon>0$ such that, for every $q$ and $g \in G, g$ appears in $B_{q}$ at at least $\varepsilon v\left(\bar{n}_{q}\right)$ positions.

Notice that the centralizer $\mathcal{C}\left(X_{x}, \mathbb{T}\right)$ of a Morse $\mathbb{Z}^{2}$-action satisfying the condition of Fact 1 always contains the transformations $\sigma_{g}(g \in G)$ defined by coordinatewise multiplication by $g, \sigma_{g}(y)=y \cdot g$.

Formulation of the main result. The aim of this paper is to prove the following

ThEOREM. There exists a strictly ergodic rank-one Morse $\mathbb{Z}^{2}$-action $\left(X_{x}, \mathbb{T}\right)$ for which

$$
\left\{\sigma_{g}: g \in G, g \neq e\right\} \subset \mathcal{C}\left(X_{x}, \mathbb{T}\right) \backslash \mathrm{Wcl}(\mathbb{T}) .
$$

We begin with a sketch of proof. The details will be provided after the section concerning autocorrelations and their properties.

The idea of the construction is as follows: $G$ is the group of complex roots of unity of degree $r \geq 2, G=\left\{1, \xi, \xi^{2}, \ldots, \xi^{r-1}\right\}$. We find a sequence $\left(E_{q}\right)_{q \geq 1}$ of blocks, increasing in size, which satisfy three conditions:

- $E_{q}(\overline{0})=1$;

- the Hamming distance between $E_{q} \cdot g$ and $T^{\bar{k}}\left(E_{q}\right)$ is bounded from below for every $q, g$, and for $\bar{k} \neq \overline{0}$ not too large;

- the left half of each block $E_{q}$ consists of constant columns, the right half consists of constant rows (as in Figure 2).

Next we take the cyclic square block $D$ of size $(r, r)$ given by $D(i, j)=$ $\xi^{i+j}$ and we define $B_{q}$ as $D \times E_{q}$. This completes the construction. Obviously, the blocks $B_{q}$ satisfy all requirements to define a strictly ergodic Morse $\mathbb{Z}^{2}$ action (see Fact 1).

The proof of the rank-one property is based on the simple observation that the product of $D$ with a constant row (or column) produces a "strap" 
of the form $(D \cdot h D \cdot h \ldots D \cdot h)$ which, except for some small (initial and final) parts, can be covered by copies of $D$.

Suppose that $\sigma_{g}$ can be weakly approximated by some shifts $T^{\bar{k}}$. This implies that there exist $y \in X_{x}$ and $\bar{k}$ such that the Hamming distance between $y \cdot g$ and $T^{\bar{k}} y$ is very small. But $y$ is built as a concatenation of the blocks $A_{q} \cdot h(h \in G)$ and we can use $q$ so large that $\bar{k}$ is very small compared to the size of $A_{q}$. Thus, in order to obtain a contradiction, it suffices to prove that the Hamming distance between $A_{q} \cdot g$ and $T^{\bar{k}}\left(A_{q}\right)$ is uniformly bounded from below for all $q, g$, and for $\bar{k} \neq \overline{0}$ not too large. At this moment the second property of $E_{q}$ will be used. As a tool allowing uniform control of the Hamming distance between a block multiplied by some $g$ and the same block shifted by some vector $\bar{k}$ we will use the so called autocorrelations. The main advantage of this parameter is that there exists a convenient estimate for autocorrelations of a product of blocks in terms of autocorrelations of the factors. For clarity, some objects appearing in the construction are visualised in figures.

Autocorrelations of two-dimensional blocks. Let $B$ be a block of size $\bar{n}$, and pick some $\bar{k} \in[-\bar{n}+\overline{1}, \bar{n}-\overline{1}]$. Define

$$
\mathbb{P}_{B}(\bar{k})=[\overline{0}, \bar{n}-\overline{1}] \cap[-\bar{k}, \bar{n}-\bar{k}-\overline{1}]
$$

(the overlap of the domain of $B$ with the domain of $T^{\bar{k}}(B)$ ). Notice that $\# \mathbb{P}_{B}(\bar{k})=v(\bar{n}-|\bar{k}|)$ (where $\left.|\bar{k}|=\left(\left|k_{1}\right|,\left|k_{2}\right|\right)\right)$. It is easy to see that if $|\bar{k}| \leq$ $\bar{n} / 2$ then $\# \mathbb{P}_{B}(\bar{k}) \geq \frac{1}{4} v(B)$, while in the opposite case $\# \mathbb{P}_{B}(\bar{k})<\frac{1}{2} v(B)$.

From now on we will assume that $G$ is the cyclic group of complex roots of unity of degree $r \geq 2, G=\left\{1, \xi, \xi^{2}, \ldots, \xi^{r-1}\right\}$.

Definition. The normalized autocorrelation of $B$ at distance $\bar{k}$ is defined as

$$
\Psi_{B}(\bar{k})=\frac{1}{\# \mathbb{P}_{B}(\bar{k})} \sum_{\bar{i} \in \mathbb{P}_{B}(\bar{k})} B(\bar{i}+\bar{k})(B(\bar{i}))^{-1} .
$$

The following fact is derived directly from the definitions of the autocorrelation and of the Hamming distance.

FACT 2. If $\left|\Psi_{B}(\bar{k})\right| \leq 1-\varrho$ then for every $g \in G$,

$$
\bar{d}\left((B \cdot g)\left[\mathbb{P}_{B}(\bar{k})\right], B\left[\mathbb{P}_{B}(\bar{k})+\bar{k}\right]\right) \geq \varrho / 2 .
$$

Proof. Suppose for some $g$ the above $\bar{d}$ distance is less than $\varrho / 2$. This means that $B(\bar{i}+\bar{k})=g B(\bar{i})$ for a fraction of at least $1-\varrho / 2$ indices $\bar{i} \in$ $\mathbb{P}_{B}(\bar{k})$. That means that the same fraction of the terms $B(\bar{i}+\bar{k})(B(\bar{i}))^{-1}$ in the sum in the definition of the autocorrelation are equal to $g$. The remaining terms of this sum lie on the unit disk, in the worst case they are all $-g$, and 
then the modulus of the autocorrelation equals

$$
\left|\left(1-\frac{\varrho}{2}\right) g+\frac{\varrho}{2}(-g)\right|=1-\varrho \text {. }
$$

Now, consider two arbitrary blocks $B$ and $C$ over $G$, and let $\bar{n}$ and $\bar{m}$ denote their respective sizes. The crucial formula in our considerations concerns autocorrelations of the product $B \times C$. We will present the formula only for $\bar{k}$ with nonnegative coordinates. The remaining (three) cases can be treated by reflection with respect to one or both axes.

Pick a vector $\bar{k} \in[\overline{0}, \bar{m} \bar{n}-\overline{1}]$; then

$$
\bar{k}=\bar{s}+\bar{n} \bar{t},
$$

with $\bar{s} \in[\overline{0}, \bar{n}-\overline{1}], \bar{t} \in[\overline{0}, \bar{m}-\overline{1}]$. Setting $\bar{s}^{\prime}=\left(s_{1}-n_{1}, s_{2}\right), \bar{s}^{\prime \prime}=\left(s_{1}, s_{2}-n_{2}\right)$, $\bar{s}^{\prime \prime \prime}=\left(s_{1}-n_{1}, s_{2}-n_{2}\right)$ we have the following four representations of $\bar{k}$ :

$$
\bar{s}+\bar{n} \bar{t}, \quad \bar{s}^{\prime}+\bar{n}(\bar{t}+(1,0)), \quad \bar{s}^{\prime \prime}+\bar{n}(\bar{t}+(0,1)), \quad \bar{s}^{\prime \prime \prime}+\bar{n}(\bar{t}+(1,1)) .
$$

The overlap area $\mathbb{P}_{B \times C}(\bar{k})$ splits into small rectangles of four types of shapes: $\mathbb{P}_{B}(\bar{s}), \mathbb{P}_{B}\left(\bar{s}^{\prime}\right), \mathbb{P}_{B}\left(\bar{s}^{\prime \prime}\right)$, and $\mathbb{P}_{B}\left(\bar{s}^{\prime \prime \prime}\right)$. The number of times each type occurs

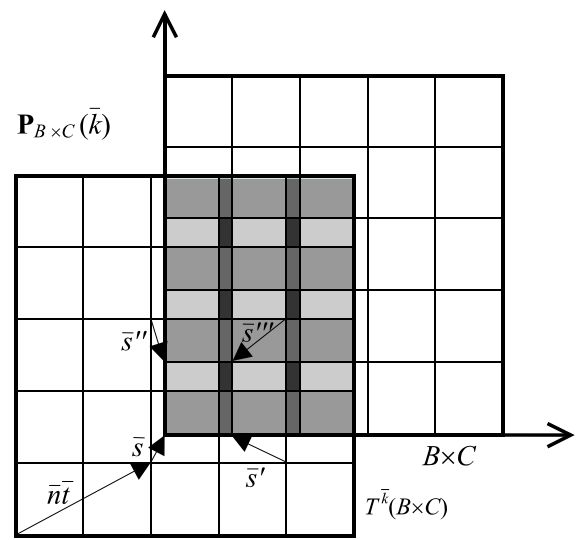

Fig. 1

is $\# \mathbb{P}_{C}(\bar{t}), \# \mathbb{P}_{C}(\bar{t}+(1,0)), \# \mathbb{P}_{C}(\bar{t}+(0,1))$, and $\# \mathbb{P}_{C}(\bar{t}+(1,1))$, respectively (see Figure 1). Applying simple arithmetical operations and estimations we obtain

$$
\begin{aligned}
& \left|\Psi_{B \times C}(\bar{s}+\bar{n} \bar{t})\right| \\
& \quad \leq \alpha_{1}\left|\Psi_{B}(\bar{s})\right| \cdot\left|\Psi_{C}(\bar{t})\right|+\alpha_{2}\left|\Psi_{B}\left(\bar{s}^{\prime}\right)\right| \cdot\left|\Psi_{C}(\bar{t}+(1,0))\right| \\
& \quad+\alpha_{3}\left|\Psi_{B}\left(\bar{s}^{\prime \prime}\right)\right| \cdot\left|\Psi_{C}(\bar{t}+(0,1))\right|+\alpha_{4}\left|\Psi_{B}\left(\bar{s}^{\prime \prime \prime}\right)\right| \cdot\left|\Psi_{C}(\bar{t}+(1,1))\right|,
\end{aligned}
$$

where

$$
\alpha_{1}=\frac{\# \mathbb{P}_{B}(\bar{s}) \# \mathbb{P}_{C}(\bar{t})}{\# \mathbb{P}_{B \times C}(\bar{k})}, \quad \alpha_{2}=\frac{\# \mathbb{P}_{B}\left(\bar{s}^{\prime}\right) \# \mathbb{P}_{C}(\bar{t}+(1,0))}{\# \mathbb{P}_{B \times C}(\bar{k})}
$$




$$
\alpha_{3}=\frac{\# \mathbb{P}_{B}\left(\bar{s}^{\prime \prime}\right) \# \mathbb{P}_{C}(\bar{t}+(0,1))}{\# \mathbb{P}_{B \times C}(\bar{k})}, \quad \alpha_{4}=\frac{\# \mathbb{P}_{B}\left(\bar{s}^{\prime \prime \prime}\right) \# \mathbb{P}_{C}(\bar{t}+(1,1))}{\# \mathbb{P}_{B \times C}(\bar{k})}
$$

(note that $\alpha_{1}+\alpha_{2}+\alpha_{3}+\alpha_{4}=1$ ). Notice also that for large $\bar{m}$, if $\bar{t}$ is not too large then the above coefficients are nearly equal to $\# \mathbb{P}_{B}(\bar{s}) / v(B)$, $\# \mathbb{P}_{B}\left(\bar{s}^{\prime}\right) / v(B), \# \mathbb{P}_{B}\left(\bar{s}^{\prime \prime}\right) / v(B)$, and $\# \mathbb{P}_{B}\left(\bar{s}^{\prime \prime \prime}\right) / v(B)$, respectively.

Now, for a block $B$ of size $\bar{n}$, set

\section{DeFinition.}

$$
1-\varrho(B)=\max \left\{\left|\Psi_{B}(\bar{k})\right|: \bar{k} \in[-\bar{n} / 2-\overline{1}, \bar{n} / 2+\overline{1}], \bar{k} \neq \overline{0}\right\} .
$$

The fact below is our main tool:

FACT 3. If $\bar{m}$ is sufficiently large then

$$
1-\varrho(B \times C) \leq \max \{1-\varrho(B), 1-\varrho(C) / 2.1\} .
$$

Proof. First suppose that $\bar{k} \in[\overline{0}, \bar{m} \bar{n} / 2+\overline{1}](\bar{k} \neq \overline{0})$. Then $\bar{k}=\bar{s}+\bar{n} \bar{t}$ with $\bar{s} \in[\overline{0}, \bar{n}-\overline{1}], \bar{t} \in[\overline{0}, \bar{m} / 2]$. Consider three cases:

(1) If $\bar{t} \neq \overline{0}$ then in the formula $(*)$ we see a subconvex combination of the numbers $\left|\Psi_{C}(\bar{t})\right|,\left|\Psi_{C}(\bar{t}+(1,0))\right|,\left|\Psi_{C}(\bar{t}+(0,1))\right|,\left|\Psi_{C}(\bar{t}+(1,1))\right|$, all of them not larger than $1-\varrho(C)$.

(2) If $\bar{t}=\overline{0}$ and $\bar{s} \leq \bar{n} / 2+\overline{1}$ then $(*)$ can be viewed as a subconvex combination of $\left|\Psi_{B}(\bar{s})\right|,\left|\Psi_{C}(1,0)\right|,\left|\Psi_{C}(0,1)\right|$ and $\left|\Psi_{C}(1,1)\right|$, hence it yields a number not larger than the maximum of $1-\varrho(B)$ and $1-\varrho(C)$.

(3) If $\bar{t}=\overline{0}$ and not $\bar{s} \leq \bar{n} / 2+\overline{1}$ then $\alpha_{1} \leq 1 / 1.92$ (for $\bar{m}$ large enough), while the remaining three terms form a combination of $\left|\Psi_{C}(1,0)\right|,\left|\Psi_{C}(0,1)\right|$ and $\left|\Psi_{C}(1,1)\right|$, which are at most $1-\varrho(C)$ each. The total is hence estimated by $1-\varrho(C) / 2.1$.

The estimates for $\bar{k}$ in the remaining parts of $[-\bar{m} \bar{n} / 2-\overline{1}, \bar{m} \bar{n} / 2+\overline{1}]$ follow by symmetry with respect to one or both axes.

We shall also need a general statement concerning the existence of long one-dimensional blocks with small autocorrelations. Let $B$ be a one-dimensional block of length $n$. We define

$$
\begin{aligned}
1-\varrho(B) & =\max _{0<k \leq n / 2+1}\left|\frac{1}{n-k} \sum_{i=0}^{n-k-1} B(i+k)(B(i))^{-1}\right|, \\
1-\varrho_{0}(B) & =\max _{0<k \leq n / 2}\left|\frac{1}{n-k} \sum_{i=k}^{n-1} B(i)\right| .
\end{aligned}
$$

FACT 4. For any $0<\varrho<1$ and each sufficiently large $n$ there exists a one-dimensional block $B$ over $G$ of length $n$ such that

(a) $1-\varrho(B) \leq 1-\varrho$,

(b) $1-\varrho_{0}(B) \leq 1-\varrho$. 
Proof. Consider an i.i.d. sequence $X_{1}, X_{2}, \ldots$ of random variables taking values in $G$ (viewed as a subset of the complex plane) with the uniform distribution $(1 / r, \ldots, 1 / r)$. Note that for any natural $k$ the sequence $X_{i+k} X_{i}^{-1}$ $(i=1,2, \ldots)$ has the same properties. Applying Hoeffding's inequality (separately to the real and imaginary parts; see e.g. [P, pp. 191-192]) we obtain

$$
P\left(\left|\frac{1}{n-k} \sum_{i=0}^{n-k-1} X_{i+k} X_{i}^{-1}\right|>1-\varrho\right) \leq 2 e^{-(n-k)(1-\varrho)^{2} / 2},
$$

Summing over $k=1,2, \ldots,[n / 2]+1$ we can write

$$
\begin{aligned}
P\left(\max _{0<k \leq n / 2+1}\left|\frac{1}{n-k} \sum_{i=0}^{n-k-1} X_{i+k} X_{i}^{-1}\right|\right. & >1-\varrho) \\
& \leq 2(n / 2+1) e^{-(n / 2-1)(1-\varrho)^{2} / 2},
\end{aligned}
$$

which is smaller than $1 / 2$ for sufficiently large $n$. Thus the set of $G$-valued blocks of length $n$ satisfying (a) is of probability larger than $1 / 2$. Analogously, the same applies to the set of blocks satisfying (b). Hence the intersection of these two sets is nonempty.

Proof of the main result. We are in a position to start the construction of our example.

Proof of the Theorem. Fix some $0<\varrho<1$. Pick an increasing sequence of natural numbers $n_{q}$. For each $q$ pick two one-dimensional blocks, $L_{q}^{\prime}$ of length $n_{q}$ and $R_{q}^{\prime}$ of length $2 n_{q}$, as specified in Fact 4 (we assume that each $n_{q}$ is sufficiently large). Clearly, we can arrange that $L_{q}^{\prime}$ starts with the symbol 1 . Now let $L_{q}$ denote the two-dimensional block of size $\left(n_{q}, 2 n_{q}\right)$ in which every row is $L_{q}^{\prime}$ (and there are $2 n_{q}$ such rows), and let $R_{q}$ be the block of the same size as $L_{q}$ in which every column is $R_{q}^{\prime}$ (and there are $n_{q}$ such columns). Notice that the columns of $L_{q}$ as well as the rows of $R_{q}$ are constant. Let $E_{q}$ denote the square block (of size $\left(2 n_{q}, 2 n_{q}\right)$ ) which is the concatenation of $L_{q}$ (on the left) and $R_{q}$ (on the right). (The structure of

\begin{tabular}{|lllll|l|l|l|l|l|}
\hline 0 & 1 & 1 & 3 & 2 & 1 & 1 & 1 & 1 & 1 \\
\hline 0 & 1 & 1 & 3 & 2 & 3 & 3 & 3 & 3 & 3 \\
0 & 1 & 1 & 3 & 2 & 0 & 0 & 0 & 0 & 0 \\
0 & 1 & 1 & 3 & 2 & 2 & 2 & 2 & 2 & 2 \\
\hline 0 & 1 & 1 & 3 & 2 & 2 & 2 & 2 & 2 & 2 \\
\hline 0 & 1 & 1 & 3 & 2 & 1 & 1 & 1 & 1 & 1 \\
\hline 0 & 1 & 1 & 3 & 2 & 3 & 3 & 3 & 3 & 3 \\
0 & 1 & 1 & 3 & 2 & 3 & 3 & 3 & 3 & 3 \\
\hline 0 & 1 & 1 & 3 & 2 & 1 & 1 & 1 & 1 & 1 \\
0 & 1 & 1 & 3 & 2 & 0 & 0 & 0 & 0 & 0 \\
\hline
\end{tabular}

Fig. 2. $E_{q}$ 
$E_{q}$ for $r=4, n_{q}=5$ is visualized in Figure 2; the integers represent the exponents $i$ of $\xi^{i}$.) We claim that

$$
1-\varrho\left(E_{q}\right) \leq 1-\frac{\varrho}{3.1} .
$$

Indeed, since $\bar{n}\left(E_{q}\right) / 2=\left(n_{q}, n_{q}\right)$ we need to consider $\bar{k} \in\left[\left(-n_{q}-1,-n_{q}-1\right)\right.$, $\left.\left(n_{q}+1, n_{q}+1\right)\right], \bar{k} \neq \overline{0}$.

Consider separately three cases (shown in Figure 3):
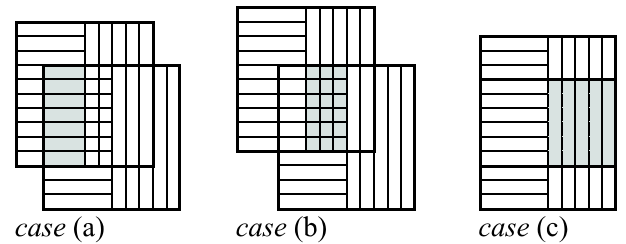

Fig. 3

(a) $\left|k_{1}\right| \leq n_{q} / 2+1, k_{1} \neq 0$. Then look at the rows of the overlap area $\mathbb{P}_{E_{q}}(\bar{k})$. The sum (as in the definition of the autocorrelation) over every such row contains the autocorrelation of $L_{q}^{\prime}$ at distance $k_{1}$ with a coefficient not smaller than $1 / 3.1$ (for sufficiently large $n_{q}$ ). Thus, the absolute value of the entire sum is at most $1-\varrho / 3.1$.

(b) $n_{q} / 2+1<\left|k_{1}\right| \leq n_{q}+1$. Then the sum over each row contains the sum of the elements of $L_{q}^{\prime}$ as in the definition of $\varrho_{0}\left(L_{q}^{\prime}\right)$ (multiplied by a constant element $g$ ), also with a coefficient of at least $1 / 3.1$. Thus the same estimate as in case (a) applies.

(c) $k_{1}=0, k_{2} \neq 0$. Then we look at the columns. Half of them contain the autocorrelation of $R_{q}^{\prime}$ at distance $k_{2}$ with $\left|k_{2}\right| \leq 2 n_{q} / 2+1$, hence not larger (in absolute value) than $1-\varrho$. In such a case the entire autocorrelation is estimated by $1-\varrho / 2$, and the claim is proved.

Let $D$ denote the square block of size $(r, r)$ defined by $D(i, j)=\xi^{i+j}$. (This definition does not depend on $q$.) We let

$$
B_{q}=D \times E_{q} .
$$

(The structure of $B_{q}$ is shown in Figure 4.) We need to estimate $1-\varrho\left(B_{q}\right)$. Since $1-\varrho(D)=1$, we cannot use Fact 3 directly. We need to go back to its proof (now $\bar{n}=(r, r)$ ). Let $\bar{k}=\bar{s}+\bar{n} \bar{t}$. If $\bar{t} \neq \overline{0}$ then part (1) of the proof of Fact 3 applies and the autocorrelation is estimated by $1-\varrho\left(E_{q}\right)$. If $\bar{t}=\overline{0}$ then $\bar{s} \neq \overline{0}$, which means that at least one coordinate of $\bar{s}$ is at least 1 , hence the size of $\mathbb{P}_{\bar{s}}(D)$ is at most $((r-1) / r) v(D)$. Thus, in $(*)$, the coefficient $\alpha_{1}$ is at most $r /(r+1)$ (assuming that $2 \bar{n}_{q}$ is large enough). As in part (3), this yields the bound $1-\varrho\left(E_{q}\right) /(r+1)$. We have proved that

$$
1-\varrho\left(B_{q}\right) \leq 1-\frac{\varrho}{3.1(r+1)} .
$$




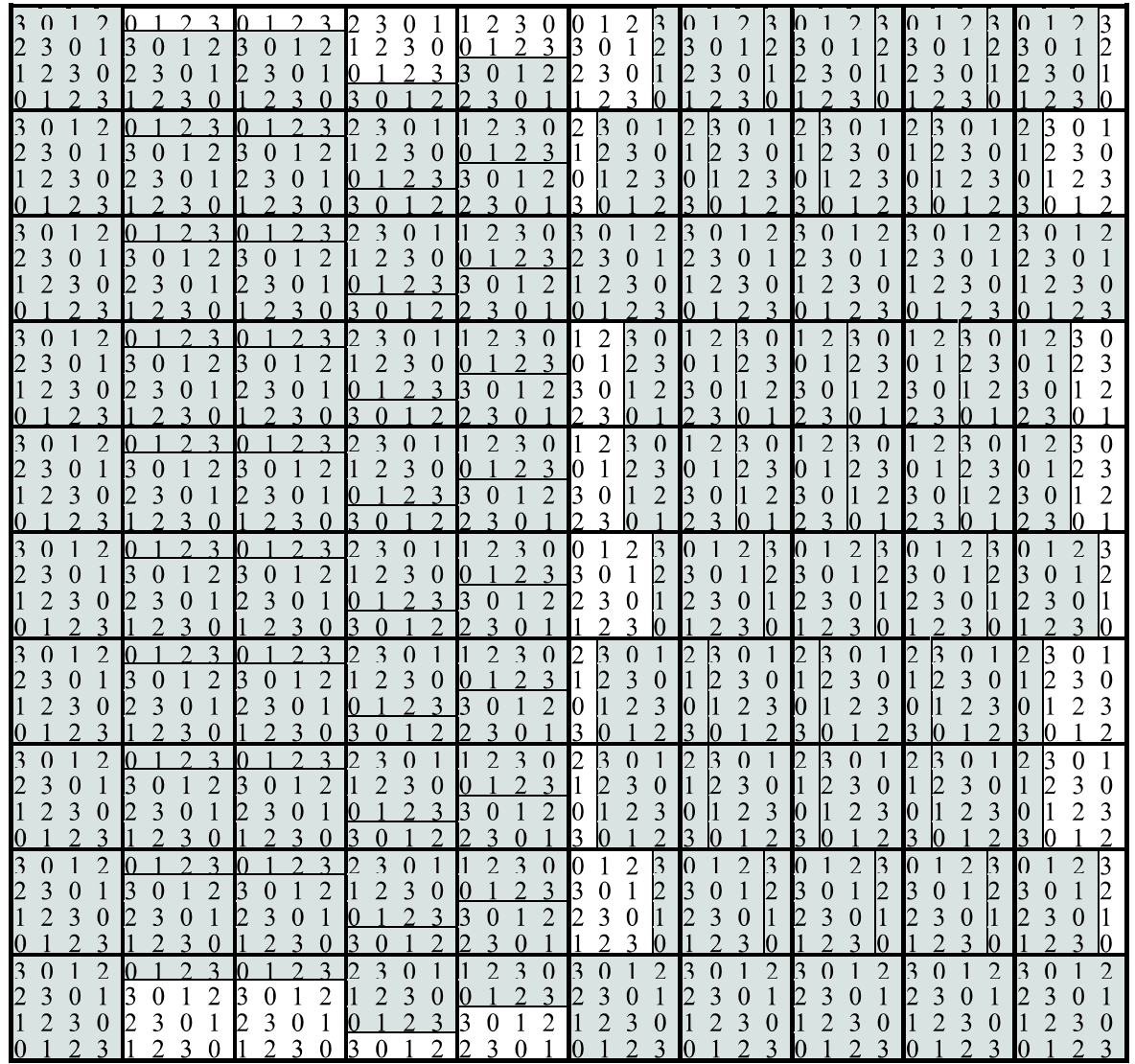

Fig. 4. $B_{q}=D \times E_{q}$

By recursive application of Fact 3 , we find that $1-\varrho\left(A_{q}\right) \leq 1-\varrho /(7(r+1))$ for every $q$ (recall that $A_{q}=B_{1} \times \ldots \times B_{q}$ and its size is $\bar{p}_{q}$ ). By Fact 2 , for any $\bar{k} \in\left[-\bar{p}_{q} / 2-\overline{1}, \bar{p}_{q} / 2+\overline{1}\right](k \neq \overline{0})$ and any $g \in G$ the $\bar{d}$-distance between $A_{q} \cdot g$ and $T^{\bar{k}} A_{q}$ (both blocks restricted to the overlap area) is not smaller than $\varrho /(14(r+1))$. For fixed $\bar{k} \in \mathbb{Z}^{2}$ with $\bar{k} \neq \overline{0}$ every element $y \in X_{x}$ can be represented as an infinite concatenation of the blocks $A_{q} \cdot h$, with $q$ large enough, so that $v\left(\bar{p}_{q}-|\bar{k}|\right) / v\left(\bar{p}_{q}\right) \geq 14 / 15$. Thus $\bar{d}\left(\sigma_{g}(y), T^{\bar{k}}(y)\right) \geq \varrho /(15(r+1))$. We have proved that none of the maps $\sigma_{g}(g \neq 1)$ belongs to $\operatorname{Wcl}(\mathbb{T})$.

It remains to show that the above described Morse $\mathbb{Z}^{2}$-action is of rank one. We will first show that for every $q$ each of the blocks $B_{q} \cdot g(g \in G)$ can be $1 / n_{q}$-covered by the block $D$. Indeed, the right half $B_{q} \cdot g$ is $D \times R_{q} \cdot g$, and since the rows of $R_{q} \cdot g$ are constant, $D \times R_{q} \cdot g$ is built of $2 n_{q}$ horizontal "straps" of the form $(D \cdot h \ldots D \cdot h)\left(n_{q}\right.$ repetitions, $\left.h \in G\right)$. But after removing a number of initial and terminal columns ( $r$ columns together $)$, 
$(D \cdot h \ldots D \cdot h)$ coincides with $(D \ldots D)\left(n_{q}-1\right.$ repetitions $)$, hence the "strap" can be $r /\left(r n_{q}\right)$-covered by $D$. Similarly, analyzing vertical "straps" of $D \times L_{q} \cdot g$ we see that this part can be $1 /\left(2 n_{q}\right)$-covered by $D$. (The cover of $B_{q} \cdot 1$ is shown in Figure 4.) Since every element $y \in X_{x}$ is a concatenation of the blocks $A_{q} \cdot h=A_{q-1} \times B_{q} \cdot h$, it can be $1 / n_{q}$-covered by the block $A_{q-1} \times D$. Obviously, the size of the last block escapes to infinity with growing index $q$ as required in the definition of rank one. The proof is now complete.

Final remark. We remark that our technique allows us to produce a similar example with the additional property of being rigid, i.e., such that the identity transformation can be approximated by $T^{\bar{k}}$ where $\bar{k}$ is not eventually constant $\overline{0}$. Recall that for rigid $\mathbb{Z}^{2}$-actions $\mathbb{T}$ is highly nondiscrete; namely $\mathrm{Wcl}(\mathbb{T})$ is uncountable. In the construction it suffices to take the square block $C_{q}$ of size $\left(n_{q}, n_{q}\right)$ filled entirely with the symbols $1\left(=\xi^{0}\right)$, and define $B_{q}$ as $C_{q} \times D \times E_{q}$. It is not hard to see that the estimates for autocorrelations of $B_{q}$ at distances larger than $\left(n_{q}, n_{q}\right)$ are the same as before. For small distances, however, $B_{q} \cdot g$ "fits" to $T^{\bar{k}}\left(B_{q}\right)$ but only for $g=1$. We skip the detailed verification.

\section{References}

[C] R. V. Chacon, Approximation and spectral multiplicity, in: Contributions to Ergodic Theory and Probability (Columbus, OH, 1970), Springer, Berlin, $18-27$.

[D-K-L] T. Downarowicz, J. Kwiatkowski and Y. Lacroix, Spectral isomorphisms of Morse flows, Fund. Math. 163 (2000), 193-213.

[G-R] G. R. Goodson and V. V. Ryzhikov, Conjugations, joinings, and direct products of locally rank one dynamical systems, J. Dynam. Control Systems 3 (1997), 321-341.

[J-R] A. del Junco and D. Rudolph, On ergodic actions whose self-joinings are graphs, Ergod. Theory Dynam. Systems 7 (1987), 531-557.

[K1] J. King, The commutant is the weak closure of the powers, for rank-1 transformations, ibid. 6 (1986), 363-384.

[K2] - Joining-rank and the structure of finite rank mixing transformations, J. Anal. Math. 51 (1988), 182-227.

[P] D. Pollard, Convergence of Stochastic Processes, Springer, New York, 1984.

[R] V. V. Ryzhikov, Mixing, rank, and minimal self-joining of actions with an invariant measure, Russian Acad. Sci. Sb. Math. 75 (1993), 405-427.

Institute of Mathematics

Technical University

Wybrzeże Wyspiańskiego 27

50-370 Wrocław, Poland

E-mail: downar@im.pwr.wroc.pl
Faculty of Mathematics and Computer Science Nicholas Copernicus University Chopina $12 / 18$

87-100 Torun, Poland E-mail: jkwiat@mat.uni.torun.pl 